\title{
Identifying Mechanical Vibration Modes of a Cantilever Using Spectrally Multiplexed Bragg Gratings and Machine Learning
}

\author{
Senta L. Jantzen ${ }^{1, *}$, Jiarui Yu ${ }^{2}$, Peter G. R. Smith ${ }^{1}$ and Christopher Holmes ${ }^{1}$ \\ 1 Optoelectronics Research Centre, University of Southampton, SO17 1BJ, UK \\ 2 Information and Electronic Engineering College of Zhejiang University, Zhejiang, China \\ * Corresponding author: S.Jantzen@soton.ac.uk
}

\begin{abstract}
In this paper, we demonstrated the use of the k-Nearest Neighbor, a machine learning algorithm, to identify mechanical vibration modes of a cantilever beam in a frequency range between $40-300 \mathrm{~Hz}$ at an accelerations of $1.1 \pm 0.1 \mathrm{~g}$. We attached fiber Bragg gratings to the cantilever structure and analyzed the spectral response during vibration. We observe small increases in spectral bandwidth of three Bragg gratings to perform a 3dimensional classification environment and evaluated the accuracy of the algorithm with independent testing data. (c) 2020 The Author(s)
\end{abstract}

\section{Introduction}

Machine learning is the ability of a system to learn from experience and to make decisions without being specifically programmed. These algorithms are often used for condition monitoring of mechanical systems to forecast time of failure, which allows scheduled maintenance and prevent unexpected machinery break downs or structural damage [1], [2].

In this work, we explore the use of a machine learning algorithm to identify the mechanical vibration modes of a cantilever with fibre Bragg gratings (FBGs) attached (see Fig. 1). FBGs are often used for structural health monitoring, since they can be implemented as quasi distributed sensors. The optical spectrum of these sensors changes with temperature and stain, but it can be difficult to detect relatively small changes in the spectrum. Machine learning algorithms can help to not only detect but also classify even small changes in the spectrum. This is particularly important for condition monitoring, to identify small changes, indicating the development of a fault in a system. Oftentimes, these changes are compared against a threshold value. Using machine learning can improve the fault prediction due to a more intelligent data-rich analysis [3].

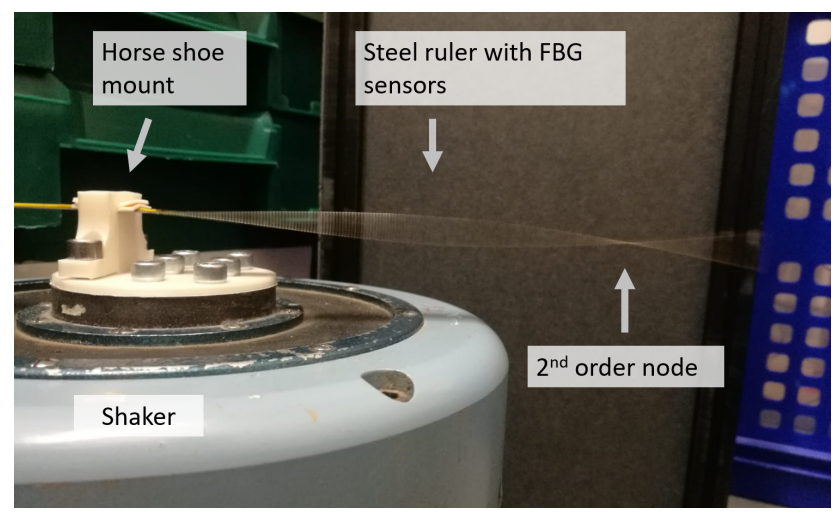

Fig. 1. Steel ruler with attached fiber Bragg gratings mounted as cantilever on a shaker, showing the cantilever driven at the second order mode at higher acceleration amplitude.

We used the k-Nearest Neighbor ( $\mathrm{kNN}$ ) algorithm to identify the mechanical modes. This algorithm relies on a training data set, where data points cluster for different events. Later recorded testing data can then be associated with respective clusters depending on the proximity to neighboring data points. The findings from this work could be used to predict potential failure of architectural structures for embedded sensors. 


\section{Experimental Procedure and Results}

A $300 \mathrm{~mm}$ long fibre with 28 Bragg gratings (each $5 \mathrm{~mm}$ in length) was placed on a steel ruler, which was clamped one sided and placed on a shaker (Derritron VP4). The shaker was driven at frequencies from 40 to $300 \mathrm{~Hz}$ with an acceleration of $1.1 \pm 0.1 \mathrm{~g}$. The resonant modes were calculated to be occurring at $52 \mathrm{~Hz}$ for the second, $146 \mathrm{~Hz}$ for the third and $287 \mathrm{~Hz}$ for the fourth order mode.

To produce a training dataset to train the algorithm, a total set of 50 optical spectra were collected for each of the four cases: no vibration, second, third and fourth order mode. The spectra were taken by connecting a broadband light source via a $3 \mathrm{~dB}$ coupler to an optical spectrum analyzer and the FBG sensor. The optical response of the gratings are dependent on their physical location relative to mechanical nodes as the strain will be highest around the nodes. Out of the 28 FBGs, three gratings with the largest spectral response were chosen as input for the 3-dimensional kNN algorithm. The spectral spread, a measure of bandwidth was calculated with

$$
\text { Spread }=\frac{\int R d \lambda}{\max |R|}
$$

where $\mathrm{R}$ is the reflected power in $\mathrm{mW}$ and $\lambda$ is the wavelength in $\mathrm{nm}$.
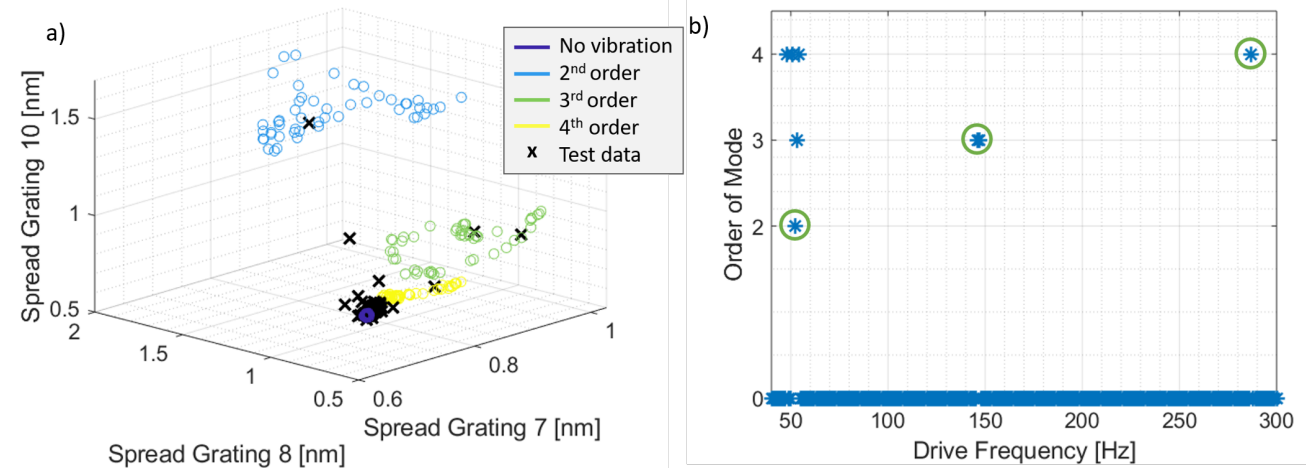

Fig. 2. a) Clustering of the training data for the four cases: "no vibration", $2^{\text {nd }}, 3^{\text {rd }}, 4^{\text {th }}$ order mode. b) The classification result for the $\mathrm{kNN}$ algorithm with $\mathrm{k}=5$. The green circles indicate correct classification at 52, 146 and $287 \mathrm{~Hz}$.

The testing data was collected by sweeping the drive frequency from $40-300 \mathrm{~Hz}$ whilst an optical spectrum was collected for every drive frequency. The clustering of the training data for the different mechanical modes along with the testing data is shown in Fig. 2 a). The training data forms distinguishable clusters for the different drive modes. The classification result for 5 nearest neighbors $(k=5)$ is shown in Fig. $2 b)$. The classification is correct for higher order modes, but shows inaccuracies for lower frequencies. The inaccuracy for lower order modes could be due to a low frequency movement which continued during the optical scanning. Further measurements will be taken with increased wait times during the drive frequency sweep.

\section{Conclusion}

In this paper, we demonstrated for the first time the identification of mechanical modes of a cantilever beam with attached Bragg gratings using a k-Nearest Neighbor machine learning algorithm over a frequency range of $40-300 \mathrm{~Hz}$ for an acceleration of $1.1 \pm 0.1 \mathrm{~g}$. This approach provides a more intelligent alternative to threshold values in fault detection. The algorithm correctly classified high order modes, but was inaccurate for lower driving frequencies. We believe that these errors are due to the vibration approaching resonances that are similar to lower amplitude resonances of that order. We will further investigate the effect of varying acceleration amplitudes particularly to analyze the accuracy limits of the algorithm.

\section{References}

1. Stetco, A., Dinmohammadi, F., Zhao, X., Robu, V., Flynn, D., Barnes, M., Nenadic, G., "Machine learning methods for wind turbine condition monitoring: A review.", Renewable Energy, 133, 2019.

2. Li, S., Sun, L., "Detectability of Bridge-Structural Damage Based on Fiber-Optic Sensing through DeepConvolutional Neural Networks". Journal of Bridge Engineering, 25(4), 2020.

3. Antoniadou, I., Worden, K. "Use of a spatially adaptive thresholding method for the condition monitoring of a wind turbine gearbox", 7th European Workshop on Structural Health Monitoring, 2014. 